\title{
Glucose Uptake Is Decreased in Affected Lower Leg Muscles of Hemiparetic Persons during Level Walking
}

\author{
Naoyuki Oi, ${ }^{1,2}$ Masatoshi Itoh, ${ }^{3, *}$ Yoshiko Tobimatsu, ${ }^{1,4}$ Shinichi Konno, ${ }^{2}$ \\ Shinichi Kikuchi ${ }^{2}$ and Tsutomu Iwaya ${ }^{1,5}$ \\ ${ }^{1}$ Department of Rehabilitation Medicine for Persons with Physical Disability, Tohoku University Graduate School \\ of Medicine, Sendai, Miyagi, Japan \\ ${ }^{2}$ Department of Orthopaedic Surgery, Fukushima Medical University, Fukushima, Fukushima, Japan \\ ${ }^{3}$ Division of Cyclotron Nuclear Medicine, Cyclotron and Radioisotope Center, Tohoku University, Sendai, Miyagi, \\ Japan \\ ${ }^{4}$ National Rehabilitation Center for Persons with Disabilities, Tokorozawa, Saitama, Japan \\ ${ }^{5}$ Nagano Health and Medical University, Nagano, Nagano, Japan
}

\begin{abstract}
Stroke patients suffer from gait disturbance due to altered leg muscle actions. Many kinesiological studies have investigated muscle actions, but the metabolic activity of muscles in stroke patients remains to be investigated. We therefore evaluated energy consumption in lower extremity muscles during level walking in hemiparetic individuals. Glucose uptake was measured by positron emission tomography (PET) using ${ }^{18} \mathrm{~F}$-fluorodeoxyglucose $\left({ }^{18} \mathrm{~F}-\mathrm{FDG}\right)$ in eight hemiparetic (mean age: 56 years) and 11 healthy (mean age: 26 years) participants. Standardized uptake ratio (SUR) was computed in each muscle to express the ${ }^{18}$ F-FDG-uptake level. SUR was compared across gluteal, thigh, and lower leg muscles and across individual muscles within each muscle group. For each muscle, SUR was compared among the paretic limb of hemiparetic participants, the non-paretic limb of hemiparetic participants, and the right limb of healthy participants. In paretic limbs, mean SUR did not differ between the three muscle groups, or between individual muscles within each muscle group. SURs of paretic lower leg muscles and gluteus minimus muscle were significantly smaller than those of non-paretic limb and healthy participants $(p<$ 0.05). In the non-paretic limb of hemiparetic participants, SUR of the lower leg muscles was larger than that of the thigh muscles $(p<0.05)$. Unexpectedly, SURs of medial hamstring and posterior tibial muscles were larger in the non-paretic limb of hemiparetic participants, compared to the right limb of healthy participants $(p<0.05)$. ${ }^{18} \mathrm{~F}-\mathrm{FDG}$ PET is useful to evaluate energy consumption levels of lower extremity muscles during level walking in hemiparetic individuals.
\end{abstract}

Keywords: ${ }^{18} \mathrm{~F}$-fluorodeoxyglucose; hemiparesis; positron emission tomography; standardized uptake ratio; walking Tohoku J. Exp. Med., 2015 December, 237 (4), 307-315. C 2015 Tohoku University Medical Press

\section{Introduction}

In rehabilitation medicine, many measures have been used to evaluate the altered functional status of skeletal muscle. Electromyography (EMG) and magnetic resonance imaging (MRI) can directly detect muscle activity during performance of physical tasks such as walking. Integrated surface EMG has most commonly been used to assess muscle activity during walking (Perry 1992). Surface EMG is beneficial for analyzing the temporal processes of muscle activity, but shows some limitations. The number of muscles that can be recorded in one study session is limited and, as the intensity of muscle contraction is generally expressed relative to the intensity recorded during a maxi- mal voluntary contraction, comparing the intensity of activity across muscles is impossible. MRI is able to show images of the muscles working during walking and other exercises (Kumagai et al. 1997; Dickx et al. 2010; Yanagisawa et al. 2015). However, if muscular activity increases above a certain threshold, MRI cannot be used for quantitative evaluation of activity, because changes in the proton density cannot be depicted.

Positron emission tomography (PET) can be used to detect the muscular uptake of a radioactive glucose analogue, ${ }^{18} \mathrm{~F}$-fluorodeoxyglucose $\left({ }^{18} \mathrm{~F}-\mathrm{FDG}\right)$. This approach has recently been used to depict muscle activity during the performance of physical tasks. Although this cannot be used to record the temporal activity of muscles, the amount

Received July 29, 2015; revised and accepted October 27, 2015. Published online November 28, 2015; doi: 10.1620/tjem.237.307. Correspondence: Naoyuki Oi, Department of Orthopaedic Surgery, Fukushima Medical University, 1 Hikarigaoka, Fukushima, Fukushima 960-1295, Japan.

e-mail: spine-oi@umin.ac.jp

* Present addresses: Sendai Medical Imaging Center, Sendai, Miyagi, Japan. 
of glucose uptake in each muscle during performance of an aerobic task can be calculated and compared across muscles.

Various reports have quantified muscle activity using ${ }^{18}$ F-FDG PET (Fujimoto et al. 1996, 2003; Tashiro et al. 1999; Gondoh et al. 2009; Shimada et al. 2011). Fujimoto et al. (1996) were the first to use this method and reported muscle activity during running. Oi et al. (2003) showed that the activity of lower leg muscles was greater than that of the thigh muscles during level walking in healthy adults. However, muscle activity during level walking has not been evaluated in hemiparetic individuals using this method. The purpose of the present study was thus to use ${ }^{18} \mathrm{~F}-\mathrm{FDG}$ PET to quantify glucose uptake level (GUL) of the lower extremity muscles during level walking in hemiparetic individuals.

\section{Subjects and Methods}

\section{Study design and participants}

This study examined the GUL of muscles in hemiparetic individuals and healthy individuals, and was conducted in the Cyclotron and Radioisotope Center at Tohoku University, Sendai, Japan. Eight hemiparetic adults (six men, two women) and 11 healthy male adults participated in this study. We recruited hemiparetic adults who had discharged from rehabilitation ward of Tohoku University Hospital. The healthy male adults were volunteers who were responded to author's request. The mean age, height and weight of hemiparetic participants were 56 years (range, 34-71 years), $162 \mathrm{~cm}$ (range, 155$172 \mathrm{~cm}$ ), and $68.4 \mathrm{~kg}$ (range, 55.0-86.0 kg), respectively. In healthy participants, these values were 26 years (range, $19-56$ years), $168 \mathrm{~cm}$ (range, 161-182 cm), and $61.7 \mathrm{~kg}$ (range, 53.4-77.6 kg), respectively.

In hemiparetic participants, the affected side was the right side in five participants and the left side in three. Causes of hemiplegia were putaminal hemorrhage $(n=5)$, cerebral infarction $(n=2)$, and pontine infarction $(n=1)$. The level of neurological impairment in the paretic lower limb was Brunnstrom level III $(n=6)$ or VI $(n=2)$. No participants had a history of any ailment related to carbohydrate metabolism or respiratory or muscular disease. One participant had a femoral intertrochanteric fracture of the paretic limb after stroke. At the time of the PET study, this participant had the same walking abil- ity and walking posture as before the fracture. Four hemiparetic participants used an ankle-foot orthosis during the 15-min walk (Table 1). None of the healthy participants regularly engaged in intense sports activity. The data of healthy participants were those used in the ${ }^{18} \mathrm{~F}$ FDG PET study of lower extremity muscular activity during level walking reported by Oi et al. (2003).

\section{PET study}

All participants refrained from eating for at least $3 \mathrm{~h}$ before starting the test. They were permitted to ingest only water before the test. After 15 min of sitting on a chair, blood was drawn from cubital vein for measurement of blood glucose level and the participant was injected with about $37 \mathrm{MBq}$ of ${ }^{18} \mathrm{~F}$-FDG. The participant then walked around a figure-of-eight track (38-m circumference) at a self-selected speed for $15 \mathrm{~min}$. After $30 \mathrm{~min}$ of rest, whole-body imaging was taken with a PET camera (SET2400W; Shimadzu, Kyoto, Japan) in three-dimensional mode with the participant in the supine position. A transmission scan was taken with the PET camera in post-injection transmission mode.

Heart rate, distance walked, and number of steps taken during the 15-min walk were measured. Distance walked and number of steps taken were used to calculate average walking speed, cadence, and step length. Exercise intensity was calculated using the following formula, and expressed as a percent of heart rate reserve (HRR):

$$
\begin{aligned}
& \text { Exercise intensity }(\%)= \\
& \quad \frac{\text { Heart rate at walking }- \text { Heart rate at rest }}{(220-\text { Age in years })-\text { Heart rate at rest }} \times 100
\end{aligned}
$$

MRI study

Magnetic resonance (MR) images of the pelvis and both lower extremities were obtained within 1 month of the PET study, using Magnetom Harmony (Siemens, Munich, Germany; static magnetic field strength, $1.0 \mathrm{~T}$ ) for hemiparetic participants and Signa Profile (GE Medical Systems, Waukesha, WI, US; static magnetic field strength, $0.2 \mathrm{~T}$ ) for healthy participants. T1-weighted MR images were used to identify the pelvic and leg muscles in the ${ }^{18} \mathrm{~F}$-FDG PET images. Muscles were identified from image slices: an axial image of the pelvis ( $4 \mathrm{~cm}$ proximal to the hip joint) for identification of the gluteal muscles; an axial image of the thigh $(20 \mathrm{~cm}$ distal to the hip joint) for identification of the thigh muscles; and an axial image of the

\begin{tabular}{|c|c|c|c|c|c|c|c|c|c|c|}
\hline Case & $\begin{array}{l}\text { Age } \\
\text { (year) }\end{array}$ & Gender & $\begin{array}{l}\text { Body } \\
\text { height } \\
(\mathrm{cm})\end{array}$ & $\begin{array}{c}\text { Body } \\
\text { weight } \\
(\mathrm{kg})\end{array}$ & Stroke type & $\begin{array}{c}\text { Paretic } \\
\text { limb }\end{array}$ & $\begin{array}{c}\text { Brunnstrom } \\
\text { recovery } \\
\text { stage: } \\
\text { Lower } \\
\text { extremity }\end{array}$ & $\begin{array}{l}\text { Duration } \\
\text { after } \\
\text { stroke onset } \\
\text { (year) }\end{array}$ & $\begin{array}{l}\text { Walking } \\
\text { aids }\end{array}$ & Comments \\
\hline 1 & 58 & Female & 155 & 61.0 & Putaminal hemorrhage & Left & III & 3.5 & AFO, Cane & \\
\hline 2 & 71 & Male & 168 & 83.0 & Pontine infarction & Right & III & 4.2 & $\mathrm{AFO}$ & \\
\hline 3 & 66 & Male & 172 & 63.5 & Putaminal hemorrhage & Left & VI & 1.3 & & \\
\hline 4 & 40 & Male & 167 & 80.5 & Putaminal hemorrhage & Right & III & 1.9 & $\mathrm{AFO}$ & Myocardial infarction \\
\hline 5 & 34 & Male & 164 & 86.0 & Cerebral infarction & Left & VI & 1.3 & & Myocardial infarction \\
\hline 6 & 56 & Male & 156 & 55.0 & Cerebral infarction & Right & III & 2.8 & AFO, Cane & \\
\hline 7 & 58 & Male & 162 & 58.0 & Putaminal hemorrhage & Right & III & 2.2 & & \\
\hline 8 & 62 & Female & 155 & 60.0 & Putaminal hemorrhage & Right & III & 3.8 & Cane & Femoral trochanteric fracture \\
\hline
\end{tabular}

Table 1. Characteristics of hemiparetic participants.

AFO, ankle foot orthosis. 
lower leg $(9 \mathrm{~cm}$ distal to the knee joint) for identification of the lower leg muscles.

\section{PET image analysis}

The axial PET images of the pelvis, thigh, and lower leg were reformatted into slices with a thickness of $16 \mathrm{~mm}$ using the software for picture conversion appended to the SET2400W camera. PET and MR images corresponding to the pelvis, thigh, and lower leg were displayed on the computer monitor. Region of interest (ROI) was defined for each muscle on the PET images, referring to the muscles shown on the MR image. The gluteal muscles examined were the gluteus maximus, gluteus medius, and gluteus minimus (Fig. 1). The thigh muscles examined were the rectus femoris, vastus medialis, vastus lateralis, vastus intermedius, adductor, medial hamstring, and lateral hamstring. The lower leg muscles examined were the tibialis anterior, tibialis posterior, peroneus, medial head of the gastrocnemius, lateral head of the gastrocnemius, and soleus (Fig. 2). The ROI of the gluteal muscle group was the total ROI of the gluteus maximus, medius, and minimus muscles. Similarly, the ROI of the thigh and lower leg muscle groups was the total ROI of the muscles of the thigh and lower leg, respectively. Imaging software was used to determine the mean value of radioactivity in each ROI. The ${ }^{18} \mathrm{~F}-\mathrm{FDG}$ uptake was expressed as standardized uptake ratio (SUR), computed in each muscle using the following formula (Kubota et al. 1985):

$$
\begin{aligned}
& \text { SUR }= \\
& \frac{\text { mean count }(\mathrm{cps} / \mathrm{ml}) \times \text { body weight }(\mathrm{g})}{\text { injection dose }(\mu \mathrm{Ci}) \times \text { calibration factor }(\mathrm{cps} / \mu \mathrm{Ci}) \times 1(\mathrm{~g} / \mathrm{ml})}
\end{aligned}
$$

Additional details on the PET image analysis can be found in our previous article (Oi et al. 2003).

\section{Statistical analysis}

SUR was compared across the gluteal muscles, thigh muscles, and lower leg muscles in hemiparetic participants. SUR was also compared across individual muscles within each muscle group (gluteal, thigh, and lower leg) in hemiparetic participants. For each muscle, SUR was compared among the paretic limb of hemiparetic participants, the non-paretic limb of hemiparetic participants, and the right limb of healthy participants. All comparisons were performed using paired t-tests, independent sample t-tests and Tukey's multiple comparison procedure. The level of significance was set at 0.05 . All statistical analyses were performed using IBM SPSS Statistics version 23 software (IBM Japan, Tokyo, Japan).

Ethics

The Clinical Committee on Radioisotope Studies, Tohoku University School of Medicine approved the study protocol. The purpose, contents, and the amount of radiation exposure were explained to the hemiparetic participants, and written informed consent was obtained.

\section{Results}

The mean heart rate and standard deviation (SD) at rest was $76 \pm 12.9$ beats/min in hemiparetic participants, while it was $66 \pm 12.9$ beats $/ \mathrm{min}$ in healthy participants. The mean heart rate during walking increased to $103 \pm 12.8$ beats/min in hemiparetic participants and to $88 \pm 15.2$ beats/min in healthy participants. The mean exercise intensity during walking was $30.6 \% \pm 10.7 \%$ HRR in hemiparetic participants and $17.0 \% \pm 6.6 \%$ HRR in healthy participants. This difference was significant (independent samples t-test, $\mathrm{p}<0.01$ ). The mean plasma concentrations of glucose in the PET study were $114.6 \mathrm{mg} / \mathrm{dl}$ for hemiparetic participants and $98.0 \mathrm{mg} / \mathrm{dl}$ for healthy participants (independent samples t-test, $\mathrm{p}<0.01$ ).

The mean distance walked in 15 min was $616 \mathrm{~m}$ for hemiparetic participants and 1,102 $\mathrm{m}$ for healthy participants. The mean step lengths were $0.44 \mathrm{~m}$ for hemiparetic participants and $0.64 \mathrm{~m}$ for healthy participants, and mean cadence was 91.5 and 114.8 steps/min, respectively. For all gait parameters, a significant difference was evident between hemiparetic and healthy participants (independent samples t-tests, all $\mathrm{p}<0.01$; Table 2).

On the coronal PET image of each muscle, the uptake of ${ }^{18} \mathrm{~F}-\mathrm{FDG}$ was similar in the proximal, middle, and distal parts of the muscle; thus, the SUR in this slice level should represent total metabolic activity of the muscle. In healthy participants, the SUR of muscles on the right side of the body was used for this study (Figs. 1, 2), because SUR of each muscle on the right side of the body did not differ sig-

Table 2. Means of physiological parameters.

\begin{tabular}{lcc}
\hline & Healthy participants & Hemiparetic participants \\
\hline Age (year) & $26(11.9)$ & $56(12.6)$ \\
Body height $(\mathrm{cm})$ & $168(5.9)$ & $162(6.5)$ \\
Body weight $(\mathrm{kg})$ & $61.7(7.3)$ & $68.4(12.6)$ \\
Heart rate at rest (beats/min) & $66(12.9)$ & $76(12.9)$ \\
Heart rate during walking (beats/min) & $88(15.2)$ & $103(12.8)$ \\
Exercise intensity during walking (\% HRR) & $17.0(6.6)$ & $30.6(10.7)$ \\
Plasma glucose (mg/dl) & $98.0(12.4)$ & $114.6(12.5)$ \\
Distance walked in 15 min (m) & $1,102(151)$ & $616(238)$ \\
Cadence (steps/min) & $114.8(11.5)$ & $91.5(15.8)$ \\
Step length (m) & $0.64(0.04)$ & $0.44(0.13)$ \\
\hline
\end{tabular}

Data are mean (SD). HRR, heart rate reserve. 

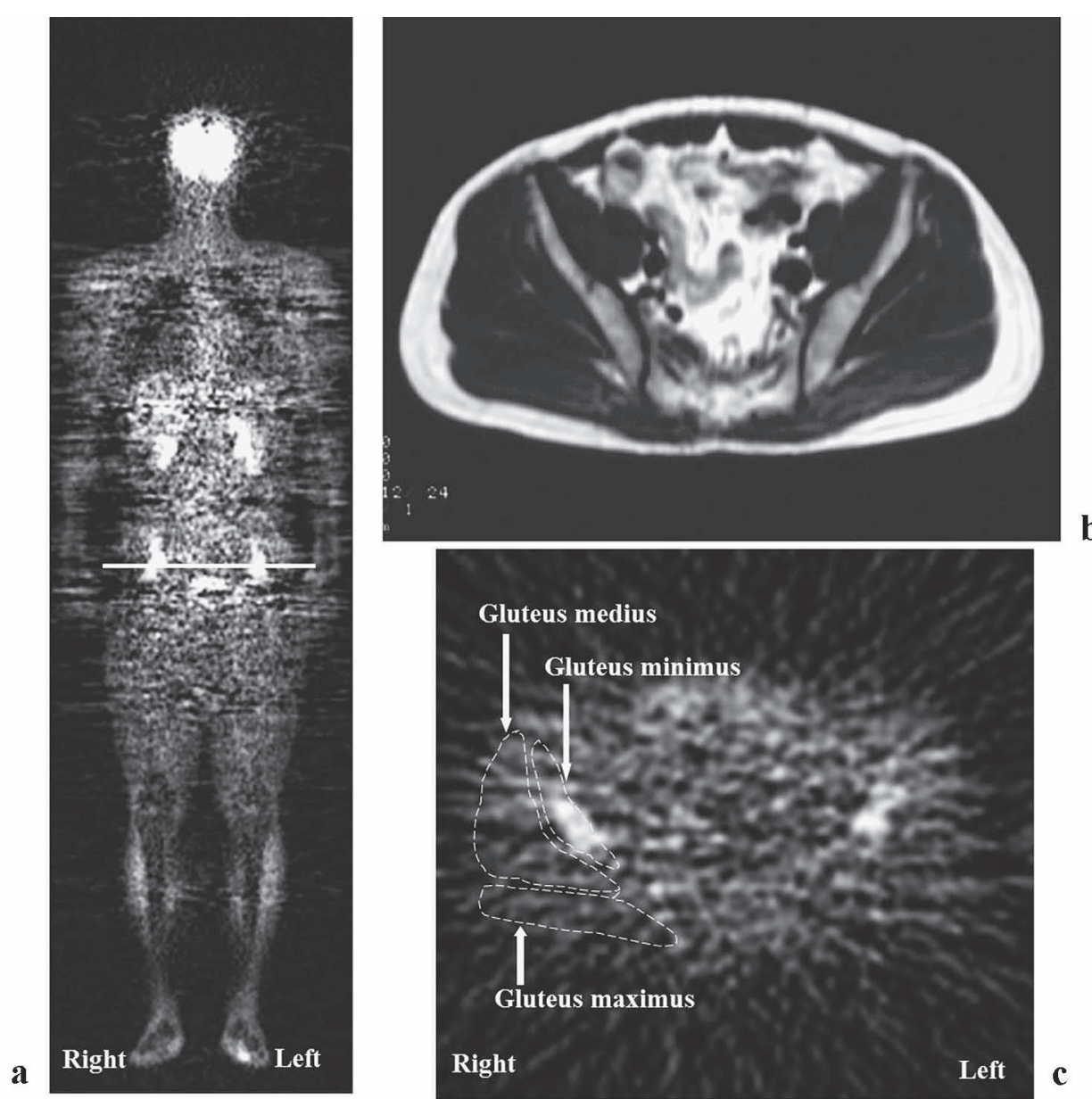

b

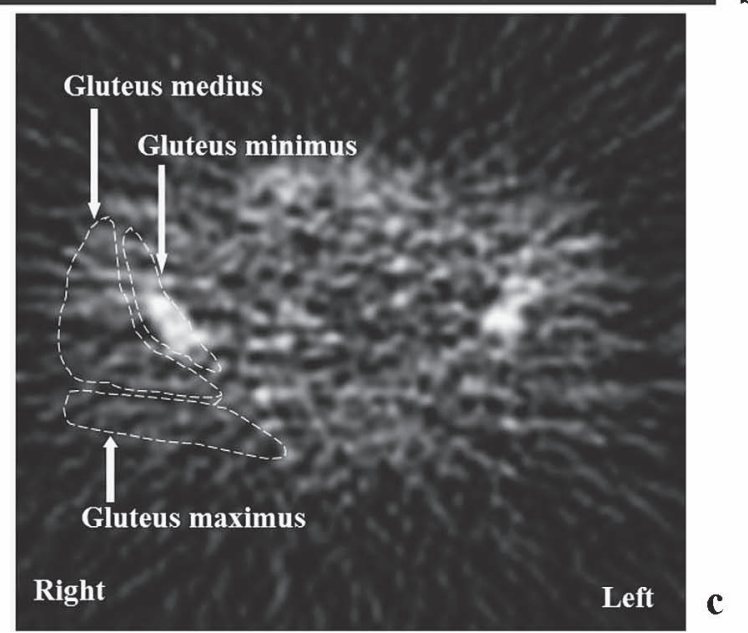

Fig. 1. Positron emission tomography (PET) of gluteal muscles in a healthy participant. These show the defined region of interest (ROI) for each gluteal muscle on PET. Radioactivity in each corresponding muscle on both sides showed no significant differences. Note the higher radioactivity, the whiter. a. Coronal PET image at $11 \mathrm{~cm}$ anterior from the back of the subject. This is an anterior-posterior view. The line shows the level of the slice in which each muscle was identified. b. Axial magnetic resonance imaging used for muscle identification. c. Axial PET image used for defining the ROI for each muscle. This is an inferior-superior view.

nificantly from that on the left side. The pattern of SUR in lower extremity muscles did not differ between hemiparetic participants using an ankle-foot orthosis and those who were not, except for the tibialis anterior muscle. As a result, no division according to the use of an ankle-foot orthosis was made in the SUR analysis.

Table 3 shows the mean SUR in each lower extremity muscle of hemiparetic and healthy participants. Fig. 3 shows PET images from a hemiparetic participant (Case 4). To clarify the age effect, we divided all participants into four age groups (19-30 years, 31-40 years, 41-65 years, and 66-71 years). We compared the mean SURs of the gluteal muscles, the thigh muscles, and the lower leg muscles, respectively, among the age groups. We found no differences of the SURs among the age group (data not shown).

In the paretic limb of hemiparetic participants, the mean SUR did not differ either between muscle groups (gluteus, thigh, and lower leg) or between individual muscles within each group. In the non-paretic limb of hemiparetic participants, the mean SUR of the lower leg muscles
$(1.40 \pm 0.61)$ was larger than that of the thigh muscles $(0.80$ \pm 0.21 ; Tukey's multiple comparison, $p<0.05$ ). In the thigh muscle group, the mean SUR of the medial hamstring muscle $(1.01 \pm 0.26)$ was larger than that of the rectus femoris muscle $(0.57 \pm 0.13$; Tukey's multiple comparison, $\mathrm{p}<$ $0.05)$. In the gluteus and lower leg muscle groups, no differences in mean SUR were identified across individual muscles.

The SUR of the lower leg muscles in the paretic limb of hemiparetic participants $(0.79 \pm 0.16)$ was significantly smaller than that of the right limb of healthy participants $(1.42 \pm 0.74$; independent samples t-test, $\mathrm{p}<0.05)$. The SURs of the soleus, anterior tibial, posterior tibial, and peroneus muscles were significantly smaller in the paretic limb of hemiparetic participants $(0.92 \pm 0.23 ; 0.79 \pm 0.53 ; 0.77 \pm$ 0.26 ; and $0.67 \pm 0.29$, respectively) than in the right limb of healthy participants $(1.71 \pm 0.99 ; 1.42 \pm 0.59 ; 1.20 \pm 0.59$; and $1.23 \pm 0.70$, respectively; independent samples t-tests, all $p<0.05$ ). Importantly, the SUR of the thigh muscles of the paretic limb of hemiparetic participants was similar to 

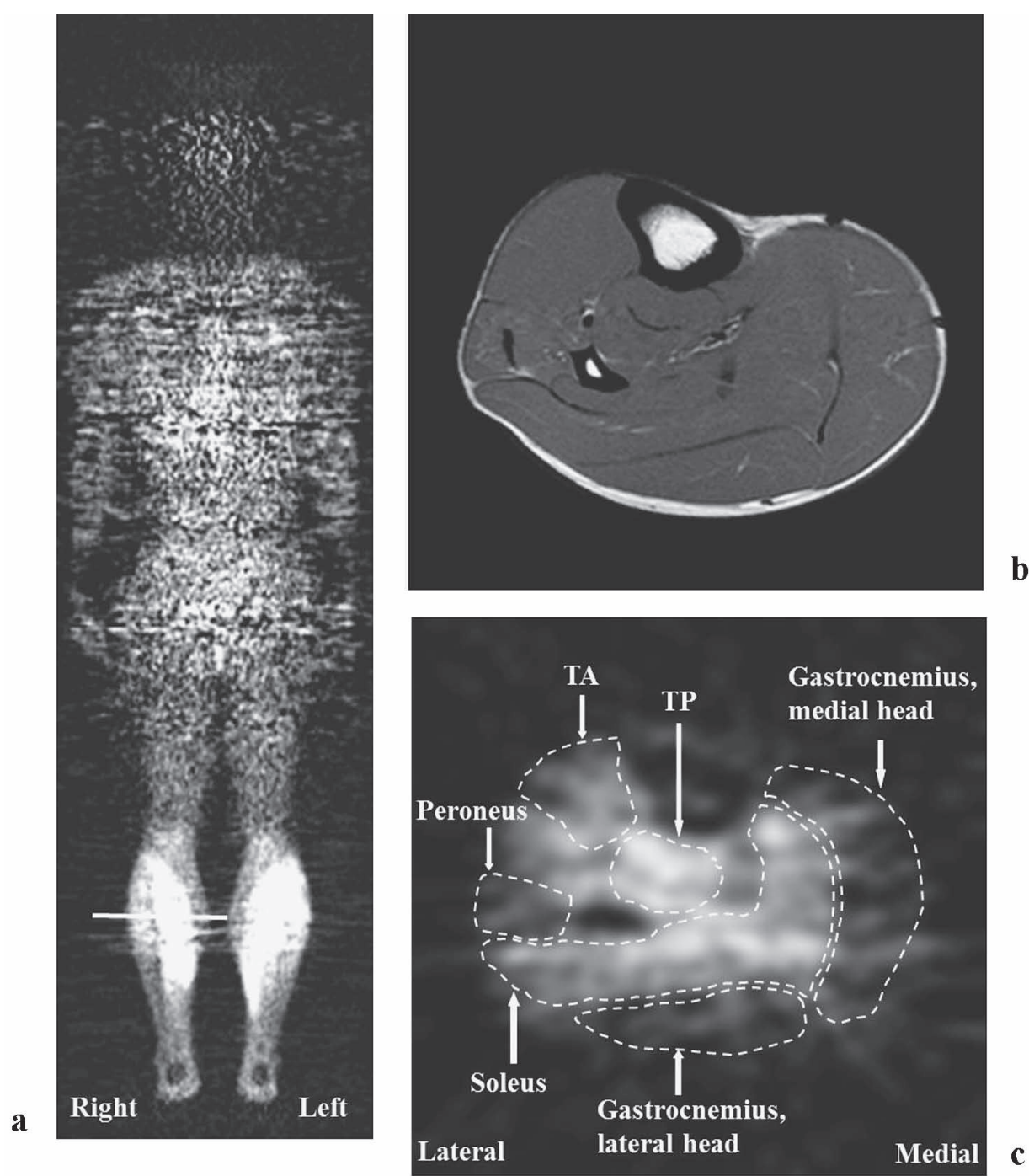

Fig. 2. PET images of lower leg muscles in a healthy participant. These show definitions for ROIs in each lower leg muscle on PET images. a. Coronal PET image at $6 \mathrm{~cm}$ anterior from the back of the subject. This is an anterior-posterior view. The line shows the level of the slice in which each muscle was identified. b. Axial magnetic resonance image used for muscle identification. c. Axial PET image (Right lower leg) used for defining the ROI for each muscle. This is an inferior-superior view.

TA, anterior tibial muscle; TP, posterior tibial muscle.

that of the right limb of healthy participants. The SUR of the gluteus minimus muscle was significantly smaller in the paretic limb of hemiparetic participants $(0.92 \pm 0.31)$ than in the right limb of healthy participants $(1.57 \pm 0.58$; independent samples t-test, $\mathrm{p}<0.01$ ).

Unexpectedly, the SUR of the medial hamstring and posterior tibial muscles was significantly larger in the nonparetic limb of hemiparetic participants $(1.01 \pm 0.26$ and $1.83 \pm 0.53$, respectively) than in the right limb of healthy participants $(0.70 \pm 0.30$ and $1.20 \pm 0.59$, respectively; independent samples t-tests, both $\mathrm{p}<0.05$ ).

The SUR of the gluteal muscles in the paretic limb of hemiparetic participants $(0.82 \pm 0.21)$ was significantly smaller than that of the non-paretic limb $(1.11 \pm 0.26$; paired t-test, $\mathrm{p}<0.05$ ). The SURs of the gluteus medius and minimus muscle were significantly smaller in the paretic limb of hemiparetic participants $(0.80 \pm 0.26$ and $0.92 \pm 0.31$, respectively) than in the non-paretic limb (1.20 \pm 0.37 and $1.36 \pm 0.40$, respectively; paired t-tests, both $\mathrm{p}<$ $0.05)$. The SUR of the medial hamstring muscle of the paretic limb of hemiparetic participants $(0.72 \pm 0.23)$ was significantly smaller than that of the non-paretic limb (1.01 \pm 0.26 ; paired t-test, $\mathrm{p}<0.05$ ). The SUR of the lower leg muscles in the paretic limb of hemiparetic participants $(0.79$ \pm 0.16 ) was significantly smaller than that of the nonparetic limb $(1.40 \pm 0.61$; paired t-test, $\mathrm{p}<0.05)$. The SURs of the soleus, lateral head of gastrocnemius, anterior tibial, and posterior tibial muscles were significantly smaller in the paretic limb of hemiparetic participants $(0.92$ $\pm 0.23 ; 0.62 \pm 0.13 ; 0.79 \pm 0.53$; and $0.77 \pm 0.26$, respectively) than in the non-paretic limb $(1.65 \pm 0.85 ; 0.90 \pm$ $0.27 ; 1.55 \pm 0.72$; and $1.83 \pm 0.53$, respectively; paired 
Table 3. SUR of lower limb muscles in hemiparetic and healthy participants.

\begin{tabular}{|c|c|c|c|}
\hline & \multirow{2}{*}{$\begin{array}{c}\text { Healthy participants } \\
\text { Right limb }\end{array}$} & \multicolumn{2}{|c|}{ Hemiparetic participants } \\
\hline & & Non-paretic limb & Paretic limb \\
\hline Gluteal muscles & $1.10(0.44)$ & $1.11(0.26)^{\pi}$ & $0.82(0.21)^{\pi}$ \\
\hline Gluteus maximus & $0.94(0.38)$ & $0.90(0.16)^{\dagger}$ & $0.79(0.20)$ \\
\hline Gluteus medius & $1.08(0.50)$ & $1.20(0.37)^{\uparrow}$ & $0.80(0.26)^{\curvearrowleft}$ \\
\hline Gluteus minimus & $1.57(0.58)^{\S}$ & $1.36(0.40)^{\dagger, \pi}$ & $0.92(0.31)^{\S, \pi}$ \\
\hline Thigh muscles & $0.70(0.24)$ & $0.80(0.21)^{*}$ & $0.79(0.19)$ \\
\hline Rectus femoris & $0.55(0.15)$ & $0.57(0.13)^{\ddagger}$ & $0.60(0.13)$ \\
\hline Vastus medialis & $0.75(0.26)$ & $0.85(0.37)$ & $0.90(0.34)$ \\
\hline Vastus lateralis & $0.56(0.22)$ & $0.66(0.29)$ & $0.73(0.18)$ \\
\hline Vastus intermeius & $0.76(0.26)$ & $0.87(0.35)$ & $0.90(0.32)$ \\
\hline Adductor & $0.81(0.30)$ & $0.84(0.24)$ & $0.83(0.24)$ \\
\hline Medial hamstring & $0.70(0.30)^{1}$ & $1.01(0.26)^{ \pm, \|, \oplus}$ & $0.72(0.23)^{\llbracket}$ \\
\hline Lateral hamstring & $0.62(0.23)$ & $0.68(0.28)$ & $0.65(0.31)$ \\
\hline Lower leg muscles & $1.42(0.74)^{\S}$ & $1.40(0.61)^{*, \pi}$ & $0.79(0.16)^{\S, \uparrow}$ \\
\hline Anterior tibial & $1.42(0.59)^{\S}$ & $1.55(0.72)^{\natural}$ & $0.79(0.53)^{\S, \mathbb{ा}}$ \\
\hline Posterior tibial & $1.20(0.59)^{\S, 1}$ & $1.83(0.53)^{\mathrm{l}, \boldsymbol{ा}}$ & $0.77(0.26)^{\S, \pi}$ \\
\hline Peroneus & $1.23(0.70)^{\S}$ & $1.43(0.88)$ & $0.67(0.29)^{\S}$ \\
\hline Gastrocnemius, medial head & $1.10(0.62)$ & $0.91(0.38)$ & $0.68(0.16)$ \\
\hline Gastrocnemius, lateral head & $0.92(0.49)$ & $0.90(0.27)^{\uparrow}$ & $0.62(0.13)^{\pi}$ \\
\hline Soleus & $1.71(0.99)^{\S}$ & $1.65(0.85)^{\natural}$ & $0.92(0.23)^{\S, \emptyset}$ \\
\hline
\end{tabular}

Data are mean (SD). SUR, standardized uptake ratio.

*Significant difference across the gluteal, thigh, and lower leg muscle groups of non-paretic limb in hemiparetic participants ( $\mathrm{p}<0.05$, Tukey's multiple comparison).

'Significant difference across individual muscles within the gluteal muscle group of non-paretic limb in hemiparetic participants ( $\mathrm{p}<0.05$, Tukey's multiple comparison).

ISignificant difference across individual muscles within the thigh muscle group of non-paretic limb in hemiparetic participants ( $\mathrm{p}<0.05$, Tukey's multiple comparison).

${ }_{\$}$ Significant difference between the paretic limb of hemiparetic participants and the right limb of healthy participants ( $p<0.05$, independent samples t-test).

'Significant difference between the non-paretic limb of hemiparetic participants and the right limb of healthy participants $(\mathrm{p}<0.05$, independent samples t-test).

'Significant difference between the non-paretic limb and the paretic limb $(\mathrm{p}<0.05$, paired t-test).

t-tests, all $\mathrm{p}<0.05)$

\section{Discussion}

In muscle tissue, the uptake of ${ }^{18} \mathrm{~F}-\mathrm{FDG}$ reflects the intensity of carbohydrate metabolism (Sokoloff et al. 1977; Phelps et al. 1979). During aerobic exercise, the uptake of ${ }^{18}$ F-FDG reflects the intensity of muscle activity. In the present study, the small increase in heart rate during level walking suggested that the exercise was aerobic (Kenney 1995). The significant increase in the mean exercise intensity also indicates that the exercise was aerobic: $30.6 \% \pm$ $10.7 \%$ HRR in hemiparetic participants and $17.0 \% \pm 6.6 \%$ HRR in healthy participants. Measuring activity of the lower extremity muscles during level walking using the uptake of ${ }^{18} \mathrm{~F}$-FDG was thus appropriate. The SUR, as the relative quantitative value of radioactivity in the ROI, was used to assess the energy consumed by each muscle of the lower extremity.

The characteristics of glucose uptake in lower limb muscles during level walking by hemiparetic participants were as follows:

1. GUL of lower leg muscles was significantly decreased in the paretic limb, relative to the non-paretic limb and healthy participants.

2. GULs of the soleus, anterior tibial, posterior tibial, peroneus, and gluteus minimus muscle were significantly decreased in the paretic limb of hemiparetic participants, relative to healthy participants.

3. GULs of the soleus, anterior tibial, posterior tibial, lateral head of gastrocnemius, medial hamstring, gluteus medius, gluteus minimus muscles were significantly decreased in the paretic limb of hemiparetic participants, relative to the non-paretic limb.

4. GULs of gluteal muscles, thigh muscles and lower leg muscles in the non-paretic limb of hemiparetic participants did not differ from those in healthy participants, except for the medial hamstring and tibialis posterior muscles. 

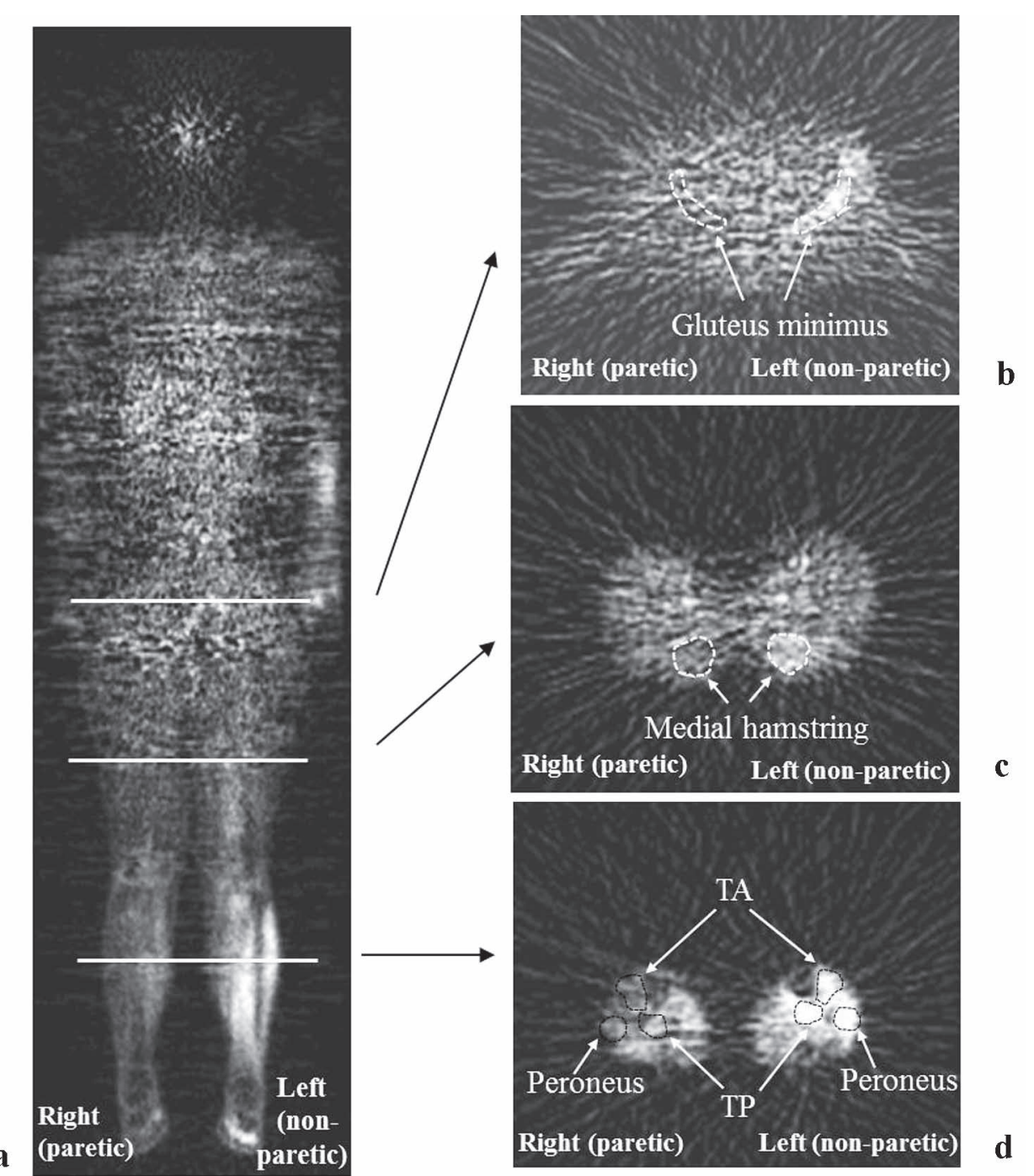

Fig. 3. PET images from a hemiparetic participant (Case 4; affected side: right). a. Coronal PET image at $6 \mathrm{~cm}$ anterior from the back of the subject, showing very low uptake of ${ }^{18} \mathrm{~F}$-fluorodeoxyglucose $\left({ }^{18} \mathrm{~F}\right.$-FDG) by muscles in the paretic (right) lower limb, and high uptake of ${ }^{18} \mathrm{~F}$-FDG by lower leg muscles in the non-paretic (left) lower limb. This is an anterior-posterior view. b. Axial PET image of the pelvis showing low uptake of ${ }^{18} \mathrm{~F}$-FDG by the gluteus minimus muscle in the paretic (right) lower limb. This is an inferior-superior view. c. Axial PET image of the thigh showing high uptake of ${ }^{18} \mathrm{~F}$-FDG by the medial hamstring muscle in the non-paretic (left) lower limb. d. Axial PET image of the lower leg showing low uptake of ${ }^{18} \mathrm{~F}$-FDG by lower leg muscles in the paretic (right) lower limb.

$\mathrm{TA}$, anterior tibial muscle; TP, posterior tibial muscle.

In the paretic limb of hemiparetic participants, GULs of the lower leg muscles (soleus, gastrocnemius, anterior tibial, posterior tibial, and peroneus) during level walking were smaller than those in the same muscles of healthy participants. Decreased activity of lower leg muscles in hemiparetic persons has previously been reported from EMG and kinematic studies. Quantitative EMG studies of the lower extremity muscles in hemiparetic individuals during level walking have shown an inability to activate the anterior tibial muscles during swing, premature calf activity prior to, or at the same time as, toe strike, and little calf activity during any period of the gait cycle, especially at push-off (Perry 1969; Kuan et al. 1999; Burridge et al. 2001). The duration of the one-legged stance phase of the gait cycle is shorter in hemiparetic individuals than in healthy individuals (Murray 1967; Peat et al. 1976). In addition, in hemiparetic persons, both the intensity of muscle activity and muscular control of the extremities are generally accepted to be more impaired in the distal limb than in the proximal part (Knutsson and Richards 1979; Knutsson 1981). Our results, which were obtained from evaluation of energy consumption, were consistent with these previously reported findings, which were obtained from EMG and kinematic studies. Decreased GUL in the lower leg muscles of the paretic limb in hemiparetic individuals may be related to impaired neurological function. Orthoses to correct ankle-foot deformities may contribute to the decreased GUL of lower leg muscles. 
Comparing activity across individual muscles is difficult in EMG studies, but ${ }^{18} \mathrm{~F}$-FDG PET can be used to compare metabolic activity across individual muscles. Our findings revealed decreased activity (in terms of energy metabolism) of the lower leg muscles compared to the thigh and gluteal muscles in the same limb, and lower activity of the lower leg muscles in the paretic limb of hemiparetic participants than in the right limb of healthy participants.

To the best of our knowledge, the present study is the first report that shows the decreased activity of the gluteus minimus muscle in the paretic limb, and increased activity of the medial hamstring and tibialis posterior muscles in the non-paretic limb of hemiparetic individuals. We found that GUL in the gluteus minimus muscle of the paretic limb in hemiparetic participants was lower than that in healthy participants. In healthy individuals, GUL in the gluteus minimus muscle was larger than that in the gluteus maximus during walking (Oi et al. 2003). Our results clearly showed that GUL during walking was decreased in the gluteus minimus muscle of the paretic limb in hemiparetic participants.

GUL of muscles in the non-paretic limb of hemiparetic participants did not differ from that in healthy participants, except for the medial hamstring and tibialis posterior muscles. Increased GULs in the medial hamstring and posterior tibial muscles may be related to abnormalities of the central nervous system resulting from the stroke, or to compensatory mechanisms related to the impaired function of the paretic limb. Our findings may be useful to analyze the mechanisms underlying altered gait patterns in hemiparetic individuals.

This study was able to demonstrate the characteristics of GUL in the muscles of the lower extremities during level walking in hemiparetic individuals using ${ }^{18} \mathrm{~F}$-FDG PET. However, some limitations to the present study must be considered. The healthy participants did not represent agematched control. The PET system we used offers a spatial resolution of $3.9 \mathrm{~mm}$ in full width at half maximum. The SUR of the small muscles may thus have contained a relatively large error. In addition, the sample population in the present study was limited to eight hemiparetic participants. A larger sample is therefore needed for further systematic investigation.

In conclusion, GULs of the lower leg muscles were significantly decreased in the paretic limb of hemiparetic participants. GULs of the soleus, anterior tibial, posterior tibial, peroneus, and gluteus minimus muscles were significantly lower in the paretic limb of hemiparetic participants than in those of healthy participants. GULs of the medial hamstring and posterior tibial muscles in the non-paretic limb of hemiparetic participants were significantly larger than in healthy participants. ${ }^{18} \mathrm{~F}$-FDG-PET is useful to evaluate energy consumption levels of lower extremity muscles during level walking in hemiparetic individuals. In addition, ${ }^{18} \mathrm{~F}$-FDG PET has the potential for use in objectively evaluating the improvement of paresis and the effectiveness of rehabilitation in hemiparetic individuals.

\section{Acknowledgments}

This work was supported in part by a Grant-in-Aid (C) 11835007 from the Ministry of Education, Culture, Sports, Science and Technology of the Japanese Government. The authors thank Shinichi Izumi, Misaki Hidaka, Naomi Yoshino, Yasuhiro Okada, Jinro Takato, Setsuko Kami, and Keiko Watanabe of the Department of Rehabilitation Medicine for Persons with Physical Disability, and the entire staff at the Cyclotron and Radioisotope Center, Tohoku University, for their support and collaboration.

\section{Conflict of Interest}

The authors declare no conflict of interest.

\section{References}

Burridge, J.H., Wood, D.E., Taylor, P.N. \& McLellan, D.L. (2001) Indices to describe different muscle activation patterns, identified during treadmill walking, in people with spastic drop-foot. Med. Eng. Phys., 23, 427-434.

Dickx, N., D’Hooge, R., Cagnie, B., Deschepper, E., Verstraete, K. \& Danneels, L. (2010) Magnetic resonance imaging and electromyography to measure lumbar back muscle activity. Spine, 35, E836-E842.

Fujimoto, T., Itoh, M., Kumano, H., Tashiro, M. \& Ido, T. (1996) Whole-body metabolic map with positron emission tomography of a man after running. Lancet, 348, 266.

Fujimoto, T., Kemppainen, J., Kalliokoski, K.K., Nuutila, P., Ito, M. \& Knuuti, J. (2003) Skeletal muscle glucose uptake response to exercise in trained and untrained men. Med. Sci. Sports Exerc., 35, 777-783.

Gondoh, Y., Tashiro, M., Itoh, M., Masud, M.M., Sensui, H., Watanuki, S., Ishii, K., Takekura, H., Nagatomi, R. \& Fujimoto, T. (2009) Evaluation of individual skeletal muscle activity by glucose uptake during pedaling exercise at different workloads using positron emission tomography. J. Appl. Physiol., 107, 599-604.

Kenney, W.L. (1995) ACSM's Guidelines for Exercise Testing and Prescription, 5th ed., Nankodo, Tokyo (Japanese version).

Knutsson, E. (1981) Gait control in hemiparesis. Scand. J. Rehabil. Med., 13, 101-108.

Knutsson, E. \& Richards, C. (1979) Different types of disturbed motor control in gait of hemiparetic patients. Brain, 102, 405-430.

Kuan, T.S., Tsou, J.Y. \& Su, F.C. (1999) Hemiplegic gait of stroke patients: the effect of using a cane. Arch. Phys. Med. Rehabil., 80, 777-784.

Kubota, K., Matsuzawa, T., Ito, M., Ito, K., Fujiwara, T., Abe, Y., Yoshioka, S., Fukuda, H., Hatazawa, J., Iwata, R., Watanuki, S. \& Ido, T. (1985) Lung tumor imaging by positron emission tomography using C-11 L-methionine. J. Nucl. Med., 26, $37-42$.

Kumagai, M., Shiba, N., Higuchi, F., Nishimura, H. \& Inoue, A. (1997) Functional evaluation of hip abductor muscles with use of magnetic resonance imaging. J. Orthop. Res., 15, 888-893.

Murray, M.P. (1967) Gait as a total pattern of movement. Am. J. Phys. Med., 46, 290-333.

Oi, N., Iwaya, T., Itoh, M., Yamaguchi, K., Tobimatsu, Y. \& Fujimoto, T. (2003) FDG-PET imaging of lower extremity muscular activity during level walking. J. Orthop. Sci., 8, 55-61.

Peat, M., Dubo, H.I., Winter, D.A., Quanbury, A.O., Steinke, T. \& Grahame, R. (1976) Electromyographic temporal analysis of gait: hemiplegic locomotion. Arch. Phys. Med. Rehabil., 57, 421-425.

Perry, J. (1969) The mechanics of walking in hemiplegia. Clin. 
Orthop. Relat. Res., 63, 23-31.

Perry, J. (1992) Gait Analysis: Normal and Pathological Function, SLACK Incorporated, Thorofare, NJ.

Phelps, M.E., Huang, S.C., Hoffman, E.J., Selin, C., Sokoloff, L. \& Kuhl, D.E. (1979) Tomographic measurement of local cerebral glucose metabolic rate in humans with (F-18)2-fluoro-2deoxy-D-glucose: validation of method. Ann. Neurol., 6, 371-388.

Shimada, H., Sturnieks, D., Endo, Y., Kimura, Y., Suzuki, T., Oda, K., Ishii, K. \& Ishiwata, K. (2011) Relationship between whole body oxygen consumption and skeletal muscle glucose metabolism during walking in older adults: FDG PET study. Aging Clin. Exp. Res., 23, 175-182.

Sokoloff, L., Reivich, M., Kennedy, C., Des Rosiers, M.H., Patlak,
C.S., Pettigrew, K.D., Sakurada, O. \& Shinohara, M. (1977) The $\left[{ }^{14} \mathrm{C}\right]$ deoxyglucose method for the measurement of local cerebral glucose utilization: theory, procedure, and normal values in the conscious and anesthetized albino rat. J. Neurochem., 28, 897-916.

Tashiro, M., Fujimoto, T., Itoh, M., Kubota, K., Fujiwara, T., Miyake, M., Watanuki, S., Horikawa, E., Sasaki, H. \& Ido, T. (1999) ${ }^{18}$ F-FDG PET imaging of muscle activity in runners. $J$. Nucl. Med., 40, 70-76.

Yanagisawa, O., Matsunaga, N., Okubo, Y. \& Kaneoka, K. (2015) Noninvasive evaluation of trunk muscle recruitment after trunk exercises using diffusion-weighted MR imaging. Magn. Reson. Med. Sci., 14, 173-181. 